\title{
Transfer RNA: A dancer between charging and mis-charging for protein biosynthesis
}

\author{
ZHOU XiaoLong \& WANG EnDuo* \\ Center for RNA Research, State Key Laboratory of Molecular Biology, Institute of Biochemistry and Cell Biology, \\ Shanghai Institutes for Biological Sciences, Chinese Academy of Sciences, Shanghai 200031, China \\ Received May 31, 2013; accepted August 13, 2013; published online August 26, 2013
}

\begin{abstract}
Transfer RNA plays a fundamental role in the protein biosynthesis as an adaptor molecule by functioning as a biological link between the genetic nucleotide sequence in the mRNA and the amino acid sequence in the protein. To perform its role in protein biosynthesis, it has to be accurately recognized by aminoacyl-tRNA synthetases (aaRSs) to generate aminoacyl-tRNAs (aa-tRNAs). The correct pairing between an amino acid with its cognate tRNA is crucial for translational quality control. Production and utilization of mis-charged tRNAs are usually detrimental for all the species, resulting in cellular dysfunctions. Correct aa-tRNAs formation is collectively controlled by aaRSs with distinct mechanisms and/or other trans-factors. However, in very limited instances, mis-charged tRNAs are intermediate for specific pathways or essential components for the translational machinery. Here, from the point of accuracy in tRNA charging, we review our understanding about the mechanism ensuring correct aa-tRNA generation. In addition, some unique mis-charged tRNA species necessary for the organism are also briefly described.
\end{abstract}

tRNA, aminoacyl-tRNA synthetase, aminoacylation, editing

Citation: Zhou X L, Wang E D. Transfer RNA: A dancer between charging and mis-charging for protein biosynthesis. Sci China Life Sci, 2013, 56: 921-932, doi: $10.1007 / \mathrm{s} 11427-013-4542-9$

The central role of tRNA is to establish genetic code by coupling an mRNA codon with an amino acid $[1,2]$. Besides this canonical function, it also carries out a wide range of non-aminoacylation functions such as cell wall biosynthesis, anti-biotic synthesis, protein degradation, bacterial membrane lipid modification, virus replication, apoptosis, amino acid biosynthesis, and precursor of small regulatory RNA, which are collectively designated non-canonical functions and have been previously reviewed elsewhere [3-8].

\section{1 tRNA aminoacylation and editing}

After maturation, tRNAs have to be first charged with cog-

*Corresponding author (email: edwang@ sibcb.ac.cn) nate amino acids by a specific group of ancient enzymes called aminoacyl-tRNA synthetases (aaRSs) to form the aminoacyl-tRNA (aa-tRNA) in the aminoacylation reaction [1,9] (Figure 1). AaRSs are divided into two classes (class I and II) on the basis of conserved sequences and characteristic structural motifs [10,11]. The aminoacylation reaction catalyzed by most aaRSs is accomplished by a two-step process [1]. Firstly, the amino acid is activated at the presence of ATP with the production of aminoacyl adenylate (aa-AMP) and the release of pyrophosphate. Secondly, one of the two hydroxyl oxygens of the $\mathrm{A}_{76}$ base of tRNAs attacks the carbonyl carbon of the aa-AMP to form the aa-tRNA with the release of the AMP. However, the aminoacylation reaction catalyzed by four aaRSs, i.e., arginyl-tRNA synthetase (ArgRS), glutaminyl-tRNA synthetase (GlnRS) and glutamyl-tRNA synthetase (GluRS), class I 


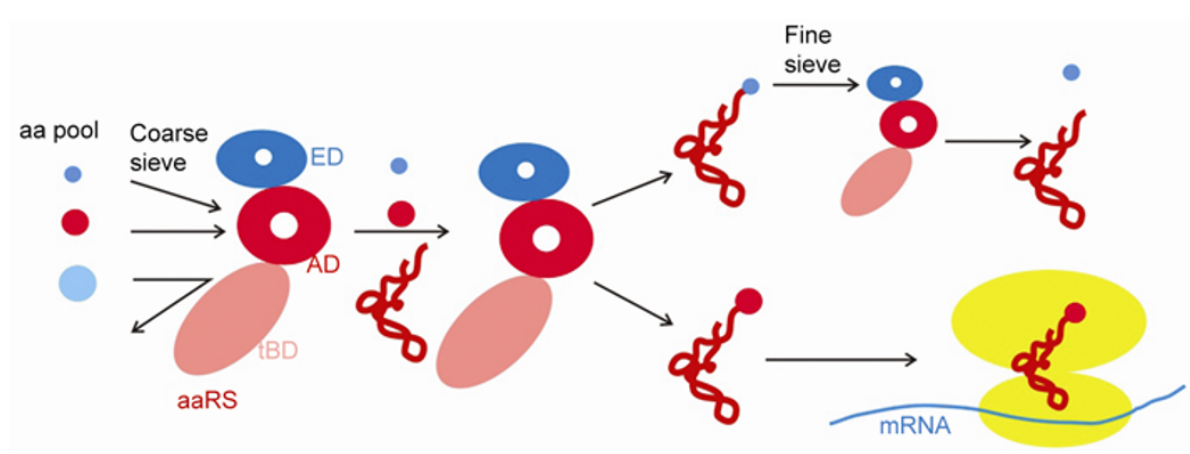

Figure 1 Aminoacylation and double-sieve mechanism by aaRSs. Amino acids larger than the cognate one are rejected by the amino acylation active site (coarse sieve); while cognate amino acid and smaller one are activated or mis-activated by aaRSs. After being transferred to cognate tRNA, the non-cognate amino acids are accommodated and hydrolyzed by the editing active site but excluded the cognate one (fine sieve). The specificity of both sieves is based on the size of different amino acids.

lysyl-tRNA synthetase (LysRS), is performed in just one step, during which the activation of the amino acid with ATP requires the presence of cognate tRNA [1].

The canonical function of aaRSs is tRNA aminoacylation to synthesize aa-tRNA pairs with adequate efficiency and accuracy, which means that the speed of the aa-tRNA production for the ribosome and the risk of yield of wrong aa-tRNA pairs should be seriously controlled and balanced [1]. The concept of "proofreading (editing)" is first experimentally described in the isoleucyl-tRNA synthetase (IleRS) system, in which addition of tRNA ${ }^{\text {Ile }}$ to the IleRS·valyladenylate complex leads to hydrolysis of the valyl adenylate rather than synthesis of Val-tRNA ${ }^{\text {Ile }}$ [12]. A "double-sieve mechanism" is proposed later to explain editing by aaRSs $[13,14]$. The active site for aminoacylation serves as the coarse sieve which binds and activates only similar or smaller amino acids at a significant rate. The active site for hydrolysis functions as the fine sieve, which eliminates the products of those amino acids that are smaller than the correct ones [15] (Figure 1). Until now, about half of the aaRSs have been found to evolve the editing capacity for removing the mis-activated amino acids and/or mis-charged tRNAs [15].

Maintaining editing activity is of great importance for cell function $[15,16]$. An editing domain with abolished or impaired hydrolysis activity would result in an ambiguous genetic code, where a single codon is not translated as a specific amino acid but rather as a statistical insertion of potentially mis-activated amino acids, leading to the statistical proteome [17]. The ambiguity derived from an editing-deficient IleRS retards cell growth at different growing conditions and makes the cell more susceptible to the ambient environment and non-cognate amino acids [18]. Overexpression of an introduced editing-deficient valyl-tRNA synthetase (ValRS), even with the background of chromosomeencoded wild-type ValRS, leads to an apoptotic response by triggering the caspase-3 pathway [19]. Strikingly, an alanine to glutamic acid mutation at a conserved site located at the editing domain of mammalian alanyl-tRNA synthetase (AlaRS) only decreases the editing activity by about $50 \%$, but causes an intracellular accumulation of mis-folded proteins in the neurons in the adult cerebellum, leading to cerebellar Purkinje cell loss and ataxia [20]. Therefore, the editing activity of aaRSs is an indispensible checkpoint for correct aa-tRNA synthesis. Any compromise of editing activity during aminoacylation of tRNAs has serious and profound negative cellular consequences, indicating the strong connection between tRNA mis-charging with cell pathology [21].

\section{Strategies employed by aaRSs to avoid tRNA mis-charging}

There are more than 20 proteinaceous amino acids and numerous amino acid analogs and metabolites in the cells. Usually several codons are evolved to specify one amino acid with several tRNA iso-acceptors recognized by an aaRS. Multiple factors including structural differences, recognition elements and anti-determinants facilitate the selection of cognate tRNAs from a large pool of similar L-shaped tRNAs by aaRSs [22]. However, selecting the cognate amino acid at an adequate level consistent with the error rate of translation is a challenge for some aaRSs because naturally existing amino acids only differ with one another at the side chain. The slight difference in size and/or chemical characteristics sometimes does not provide a sufficient recognition motif for aaRSs. Therefore, Val is misactivated by $E$. coli IleRS only 180 -fold less efficiently than Ile [23]. Similarly, Nva is obviously mis-activated by Aquifex aeolicus leucyl-tRNA synthetase (AaLeuRS) with only a 72-fold less efficiency when compared with cognate Leu [24]. To address this dilemma, aaRSs utilize several different, seemingly irrelevant but correlated editing strategies to hydrolyze mis-activated aa-AMP (pre-transfer editing) and/or aa-tRNA (post-transfer editing) products [15,25].

\section{1 tRNA-independent pre-transfer editing}

When a non-cognate amino acid is bound and mis-activated, 
the produced aa-AMP might be released from the active site into solution because of its low affinity with the enzyme compared with cognate aa-AMP. The released adenylate in the solution may undergo non-aaRS-catalytic hydrolysis due to its intrinsic instability of mixed anhydride functionality $[26,27]$. Several studies have shown that this pathway (selective release, Figure 2) only constitutes a minor and negligible contribution to the overall editing mechanism [24,26-29].

Non-cognate aa-AMP may also be hydrolyzed by the aaRS at the absence of tRNA, which is called "tRNAindependent pre-transfer editing" [24] (Figure 2). Wild-type E. coli prolyl-tRNA synthetase (EcProRS) displays a preference for Pro over non-cognate Ala about 2300-fold, which is well within the regime required for editing, implying $E c$ ProRS needs editing activity for prevention of Ala-tRNA ${ }^{\text {Pro }}$ synthesis [28]. Indeed, saturated Ala obviously stimulates the ATP hydrolysis catalyzed by $E c$ ProRS with the absence of tRNA ${ }^{\text {Pro }}$, indicating EcProRS possesses a tRNA-independent pre-transfer editing activity towards Ala. However, the EcProRS K279A mutant, deficient in post-transfer editing, accumulates a significant amount of Ala-tRNA ${ }^{\text {Pro }}$ [28]. In the LeuRS system, Nva obviously induces the tRNAindependent pre-transfer editing of $A a$ LeuRS, as measured in the AMP formation assay, which is based on the repetitive cycles of synthesis-hydrolysis of non-cognate products [24]. Quantification of various editing pathways reveals that this mechanism only contributes to about $7 \%$ of the total editing. Introduction of Ala to the conserved and essential Asp leads to an $A a$ LeuRS D373A mutant, which lacks the post-transfer editing but readily synthesizes mis-charged tRNA $^{\text {Leu }}$ [24]. tRNA-independent pre-transfer editing is also recently identified in the Saccharomyces cerevisiae threonyl-tRNA synthetase ( $S c$ ThrRS) system. It contributes to about $13 \%$ of the total editing, and mutants harboring only this editing activity fails to stop Ser-tRNA ${ }^{\text {Thr }}$ synthesis [29]. Therefore, all of the examples indicate the tRNA-independent pre-transfer editing pathway is only a minor editing mechanism employed by some aaRSs and its efficiency is insufficient to ensure translational quality control. The active site of tRNA-independent pre-transfer editing is believed to be localized in the aminoacylation active site. This is implied in the GlnRS system, which has no editing domain but has the intrinsic ability to hydrolyze Gln-AMP [26]. The editing domain-deleted mutant of EcProRS could still perform tRNA-independent pre-transfer editing [27]. E. coli and $S$. cerevisiae seryl-tRNA synthetases (SerRSs), both lacking post-transfer editing domains, also possess tRNA-independent pre-transfer editing [30]. Furthermore, recent studies of Mycoplasma mobile LeuRS (MmLeuRS) and S. cerevisiae mitochondrial ThrRS, which both naturally lack an editing domain, edit Nva-AMP and Ser-AMP in the absence of tRNA, respectively [31,32]. Thus, all lines of evidence strongly indicate tRNA-independent pre-transfer editing occurs at the aminoacylation active site. The bound noncognate aa-AMP may be sensed by some elements, and a local conformational change may be induced to allow a hydrolytically active water to cleave it. However, its detailed mechanism remains to be clarified.

\section{2 tRNA-dependent pre-transfer editing}

AaRS-catalyzed hydrolysis of non-cognate aa-AMP, triggered by the tRNA addition, is called "tRNA-dependent pre-transfer editing" [29,33] (Figure 2). Not all the aaRSs, which possess tRNA-independent pre-transfer editing, have the tRNA-dependent pre-transfer editing, implying it has no co-relation between these two mechanisms [28,31]. For example, EcProRS only harbors the tRNA-independent but not tRNA-dependent pre-transfer editing [28]. Because of the intrinsic instability of mixed anhydrides and the difficulty in separating it from post-transfer editing, the mechanism might be diverse within different systems. We tend to classify it into two categories based on the experimental data so far.

\subsubsection{Diffusion model}

As mentioned before, in IleRS system, the addition of tRNA $^{\text {Ile }}$ to the IleRS - valyl-adenylate complex leads to hydrolysis of the valyl adenylate rather than synthesis of

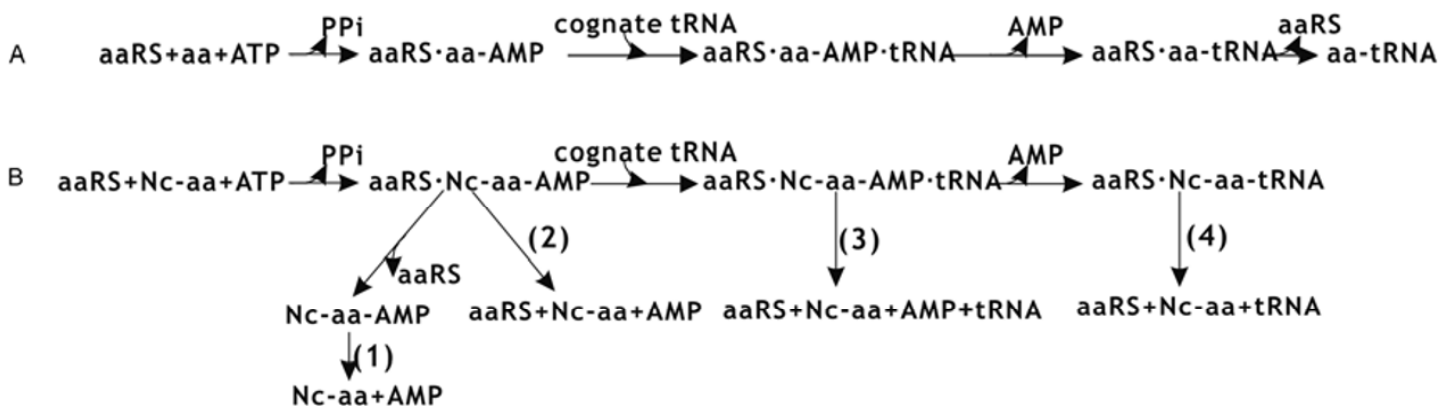

Figure 2 Schematic presentation of the aminoacylation and editing pathways. A, Cognate amino acid (aa) is activated and then transferred to cognate tRNA, generating aa-tRNA for ribosomal protein biosynthesis. B, Non-cognate amino acid (Nc-aa) is mis-activated and then edited by different mechanisms. Route 1 (selective release), route 2 (tRNA-independent pre-transfer editing), and route 3 (tRNA-dependent pre-transfer editing) belong to the pre-transfer editing pathways. Route 4 (deacylation of mis-charged tRNAs) is the post-transfer editing pathway. 
Val-tRNA $^{\text {Ile }}$ [12]. Fluorescence translocation based assay combined with structure-directed mutagenesis in the CP1 domain of IleRS indicates that the hydrolysis of Val-AMP occurs in CP1 [34,35]. Furthermore, the X-ray crystal structure shows that both the substrate of pre-transfer editing and post-transfer editing could be bound at the CP1 domain with overlapping sites in LeuRS and IleRS [36,37]. These evidences suggest that the mis-activated amino acid is translocated from the aminoacylation active site to the editing active site in a tRNA-dependent manner [38,39]. Detailed mutagenesis at the interface between editing and aminoacylation domains leads to a "post transfer initiated pre-transfer editing"/diffusion model [34]. In this model, IleRS mis-activates the non-cognate Val and transfers it the tRNA $^{\text {Ile; }}$; then the IleRS-Val-tRNA ${ }^{\text {Ile }}$ complex performs a conformational change to translocate the 3 '-end of Val-tRNA ${ }^{\mathrm{Ile}}$ from the aminoacylation active site to the editing active site about $30 \AA$ away. After an initial step of post-transfer editing, the uncharged 3 '-end stays at CP1 and induces the conformation capable of activating pre-transfer editing. The subsequently produced Val-AMP then diffuses from the aminoacylation active site to the editing active site for hydrolysis by a tRNA-dependent pre-transfer editing mechanism. A proposed channel spanning between the two editing sites, is consistently observed in both Staphylococcus aureus IleRS-tRNA ${ }^{\mathrm{Ile}}$ [39] and Thermus thermophilus ValRS-tRNA $^{\mathrm{Val}}$ [40] complexes. The opening of the KMSKS loop at the aminoacylation domain might be induced by the tRNA binding and might result in an easier release of non-cognate aa-AMPs from the aminoacylation site to the editing site [39]. The cognate aa-AMP might be bound more tightly, leading to no diffusion or rejection by the editing active site after diffusion. However, this model is not observed in the class II aaRS.

\subsubsection{Aminoacylation active site-editing model}

Studies of the GlnRS shows that a non-aminoacylated tRNA $\left(\mathrm{tRNA}^{\mathrm{Gln}} 2^{\prime} \mathrm{H}\right)$ significantly induces the pre-transfer editinglike hydrolysis of Gln-AMP [26]. GlnRS is a natural class II aaRS without a post-transfer editing domain, implying that the aminoacylation active site has the potential to hydrolyze aa-AMP. Later studies from EcLeuRS-AN2690 complex shows that despite deficiencies in post-transfer editing derived from an adduct formed between the tRNA $\mathrm{A}_{76}$ and the inhibitor in this state, EcLeuRS still has an obviously higher level of editing than tRNA-independent pre-transfer editing [33]. tRNA-dependent pre-transfer editing is also observed in AaLeuRS [33]. It occupies 35\% and 65\% of total editing in EcLeuRS and AaLeuRS, respectively. Similarly, tRNAdependent pre-transfer editing is also described in E. coli IleRS and ValRS systems [41], both supporting the hypothesis that hydrolysis occurs in the aminoacylation active site. Furthermore, after blocking post-transfer editing active site residues $\left(\mathrm{H}^{151}\right.$ and $\left.\mathrm{H}^{155}\right)$ in ScThrRS, the ScThrRS-H151A/ H155A still possesses strong tRNA-dependent pre-transfer editing, accounting for about $78 \%$ of the total editing [29]. It is notable that the post-transfer editing domain is unable to perform pre-transfer editing of Ser-AMP based on the $\mathrm{X}$-ray crystal structure [42]. This data suggest that tRNA-dependent pre-transfer editing occurs in the aminoacylation active site. Based on the evidence from class I LeuRS [33,43,44] and class II ThrRS [29] systems, the correct positioning of tRNA 3 '-end and the editing domain seems to be a prerequisite for hydrolysis in the aminoacylation active site. Introduction of both an EcLeuRS-Y330D and ScThrRS-Y291E mutant totally abolishes the tRNAdependent pre-transfer editing. Therefore, interaction of tRNA 3 '-end with the editing domain seemingly confers to the ribo-nucleoprotein a three-dimensional conformation required for the hydrolytic pre-transfer catalysis. Despite the fact that tRNA-dependent pre-transfer editing always significantly contributes to the total editing capacity [29,33], more than half in some cases, mutants only possessing pre-transfer editing obviously synthesize mis-charged tRNAs, indicating it is not an efficient translational quality control step. It may be an initial intrinsic editing pathway corresponding to a low level of aminoacylation accuracy for the ancient protein biosynthesis in the stage after the transition from a RNA world to the protein world.

Although two models have been proposed, neither gives a clear description of hydrolysis. To further study the mechanism of tRNA-dependent pre-transfer editing, it would be necessary to identify crucial elements directly and specially involved in the hydrolysis of non-cognate aa-AMP.

\section{3 (tRNA-dependent) post-transfer editing}

Post-transfer editing refers to the hydrolysis of the aminoacyl ester linkage of mis-charged tRNAs (Figure 2), which has been described in class I IleRS [45], ValRS [46], LeuRS [47] and class II ThrRS [42,48], ProRS [49], AlaRS [50] and phenylalanyl-tRNA synthetase (PheRS) [51] systems. It is the most extensively studied pathway of aminoacylation quality control and could be classified into cis-editing by aaRSs and trans-editing by some freestanding proteins on the basis of release from an aaRS [15]. Trans-editing also includes the re-capture and hydrolysis of mis-charged tRNA by an aaRS after the mis-charged tRNA is released from it [52]. This section is focused on the various aspects of cis-editing.

\subsubsection{CCA translocation}

Post-transfer editing is always involved in the translocation of the $3^{\prime}$-end of mis-charged tRNA from the aminoacylation synthetic site to the editing active site in most aaRS systems, which are usually more than $30 \AA$ apart [53,54]. A series of aaRS-tRNA co-crystal structures in either aminoacylation or editing state have captured snapshots of the translocation process [39,40,55-57]. The conformation of the main body of tRNA is thought to be unchanged during the transloca- 
tion of the 3'-CCA end between two sites. However, the recent LeuRS-tRNA ${ }^{\text {Leu }}$ complex indicates that both the tRNA and enzyme undergo significant rotation during this dynamic process [58]. Moreover, studies from AlaRStRNA $^{\text {Ala }}$ system show that the mis-charged tRNA ${ }^{\text {Ala }}$ first dissociates from the aminoacylation site and then associates with the editing active site because both domains recognize the same tRNA element [59,60]. Despite modeling from various structures, the dynamic and complete process of the aminoacylation domain to the editing domain of the mis-charged tRNA is still unclear.

\subsubsection{Domain for editing}

(i) LeuRS/IleRS/ValRS systems. Extensive studies have revealed that the class I LeuRS, IleRS and ValRS share a homologous CP1 domain for post-transfer editing [47,61-66]. The CP1 domain is inserted into the Rossmann fold aminoacylation domain and separates it into two halves [54]. It was first found to be involved in the editing reaction on the basis of its crystal structure followed by experimental data that showed isolated CP1 domains from IleRS and ValRS directly catalyze the hydrolysis of Val-tRNA ${ }^{\text {Ile }}$ or Thr-tRNA ${ }^{\mathrm{Val}}$, respectively [67]. Similarly, the CP1 domain from LeuRS is also found to be responsible for the post-transfer editing in various LeuRS systems $[47,68,69]$. Interestingly, the LeuRS CP1 domain has been proven to be an effective target for developing small molecular inhibitors, such as AN2690 which forms an adduct with the tRNA ${ }^{\text {Leu }}$ $\mathrm{A}_{76}$ in the editing active site, thus blocking Leu-tRNA ${ }^{\text {Leu }}$ synthesis [70]. In contrast, the editing domains of class II aaRSs are more evolutionarily divergent in location and sequence.

(ii) ProRS system. ProRS has been found to significantly mis-activate Ala and form Ala-tRNA ${ }^{\text {Pro }}$ [28]. In prokaryote-type ProRS, a large insertion domain (INS) between motifs 2 and 3 of the catalytic domain is responsible for deacylating Ala-tRNA ${ }^{\text {Pro }}$. An INS deletion ProRS mutant fails to hydrolyze mis-charged tRNA ${ }^{\text {Pro }}$ [49]. In addition, the INS domain is not exclusively found in eubacterial ProRSs; it is also found at the $\mathrm{N}$ terminus of the eukaryotic/archaea-like ProRSs of yeasts and single-celled parasites [71]. However, the INS domain is absent from certain prokaryote-type ProRSs, such as those of a-proteobacteria and eukaryotic organelles [71]. Thus, the editing property in even one single class II aaRS system is not conserved.

(iii) ThrRS system. ThrRS has the intrinsic ability to mis-activate non-cognate Ser. The N2 domain of bacterial and eukaryotic ThrRS performs the function of removing non-cognate Ser attached to $\operatorname{tRNA}^{\mathrm{Thr}}$ [42]. However, the editing domain of ThrRS is not conserved through evolution, as most archaeal ThrRSs possess a unique sequence at their $\mathrm{N}$-terminus $[72,73]$. This unique module shares significant structural homology with the D-aminoacyl-tRNA deacylase (DTD), which specially removes the D-amino acid, but not the L-amino acid, from a mis-charged tRNA [73]. Recent biochemical and biophysical studies reveal that this DTD-like editing domain of archaeal ThrRS could bind cognate Thr-tRNA ${ }^{\mathrm{Thr}}$, and functional positioning of substrates rather than steric exclusion is the key for the mechanism of discrimination. A properly positioned catalytic water molecule facilitates the hydrolysis of Ser-tRNA ${ }^{\text {Thr }}$ while the binding of the cognate Thr-tRNA ${ }^{\text {Thr }}$ excludes it, leading to the failure in hydrolysis of correct charged tRNA ${ }^{\mathrm{Thr}}$ [74]. This finding improves our understanding of the double-sieve mechanism. In addition, in some crenarchaeal species, the DTD-like editing domain is missing in ThrRS (ThrRS-cat). Instead, those species encode a freestanding protein (ThrRS-ed), which is not homologous to the editing domain of other ThrRSs, which specifically trans-edits Ser-tRNA $^{\mathrm{Thr}}$. ThrRS-cat cooperates with ThrRS-ed to ensure the translational quality control [48].

(iv) PheRS system. PheRS, a class II aaRS, is a tetrameric enzyme consisting of two $\alpha / \beta$ heterodimers. On the basis of the primary sequence, PheRS could be categorized into two groups, the bacterial and archaeal/eukaryal types. PheRS significantly mis-activates non-cognate Tyr and the resultant Tyr-tRNA ${ }^{\text {Phe }}$ should be removed by the posttransfer editing mechanism [75]. The $\alpha$ subunit performs the aminoacylation reaction but both subunits interact with tRNA $^{\text {Phe }}$. Biochemical and structural studies have showed that the B3/B4 domain of the $\beta$ subunit from two PheRS groups harbors the editing active site for post-transfer editing [51]. Consistently, editing defects of PheRS result in efficient mistranslation of Phe to Tyr conversion in vitro within the translation system [76].

(v) AlaRS system. AlaRS has to clear both Gly-tRNA ${ }^{\text {Ala }}$ and Ser-tRNA ${ }^{\text {Ala }}$ species. Being a class II aaRS, it consists of a N-terminal aminoacylation domain, tRNA recognition domain, editing domain, and C-terminal C-Ala domain [60]. The $\mathrm{G}_{3}-\mathrm{U}_{70}$ base pair of tRNA ${ }^{\text {Ala }}$ is the identity element for both aminoacylation and editing domains [59]. A point mutation in the editing domain of mammalian AlaRS caused an intracellular accumulation of mis-folded proteins in neurons and resulted in cerebellar Purkinje cell loss and ataxia [20].

\subsection{3 tRNA determinants for post-transfer editing}

AaRSs recognize a specific group of nucleotides for both aminoacylation and editing. The tRNA identity elements for aminoacylation have been extensively studied and identified [22]; however, knowledge is relatively limited regarding the determinants for the editing process. Usually, editing determinants for both aminoacylation and editing are highly correlated. Studies from ValRS and LeuRS systems reveal that editing determinants for two reactions are always overlapped [43,77-79]. However, the D-loop of tRNA ${ }^{\text {Ile }}$ plays a crucial role in editing but not aminoacylation in IleRS system [80]. The bases for the tertiary structure formation be-

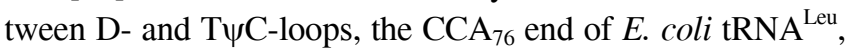
and the anti-codon arm of both A. aeolicus and $S$. cerevisiae tRNA $^{\text {Leu }}$ play an important role in editing $[43,78,79,81]$. 
AlaRS recognizes the $\mathrm{G}_{3}-\mathrm{U}_{70}$ base pair in both aminoacylation and post-transfer editing reactions but with different domains, indicating that mis-charged tRNA ${ }^{\text {Ala }}$ should first dissociate from aminoacylation domain and then be re-captured by the editing domain $[59,60]$.

\subsubsection{Mechanism}

(i) Amino acid specificity. According to the Double Sieve Mechanism, the cognate amino acid has no access to the editing active site because of steric exclusion [14]. Indeed, as shown in the LeuRS CP1 domain, a Thr residue (T252 in EcLeuRS) at the bottom of the editing pocket functions as an editing specificity determinant [82]. Manipulation of the pocket size by introducing a larger or a smaller residue at this site could artificially shift the editing specificity of post-transfer editing. A very similar mechanism is also used in the closely related IleRS and ValRS systems [63,64]. From the crystal structure, the editing active site of the N2 domain of E. coli ThrRS (EcThrRS) is just suitable for binding Ser-tRNA ${ }^{\text {Thr }}$. The pocket is too narrow to accommodate Thr, whose side chain methyl group would clash with the side chain of several conserved amino acids ( $\mathrm{His}^{77}$, $\mathrm{Tyr}^{104}$ and $\mathrm{Asp}^{180}$ ) [42]. So far, most aaRSs from both class I and class II employ similar mechanism to discriminate cognate verse non-cognate amino acids. However, some aaRSs also evolved alternative ways to ensure hydrolysis specificity. For example, the editing domain of ThrRS from Pyrococcus abyssi could accommodate cognate Thr in addition to non-cognate Gly and Ser. However, the binding of Thr-tRNA $^{\text {Thr }}$ would exclude the catalytically active water which is essential for hydrolysis [74].

(ii) Hydrolysis mechanism. The mechanism is most clearly elucidated in the EcThrRS system [42]. The EcThrRS complexed with $\mathrm{SerA}_{76}$ (mimicking the very terminus of Ser-tRNA ${ }^{\mathrm{Th}}$ ) reveals two crucial catalytic water molecules. The first water, deprotonated by $\mathrm{His}^{73}$, performs a nucleophilic attack toward the ester bond while another water molecule donates a proton to the $\mathrm{O}^{\prime}$ ' leaving group, leading to the breakage of the ester bond. A series of highly conserved residues (His ${ }^{77}$, Lys $^{156}$, Cys ${ }^{182}$, etc.) participate in the correct positioning of the substrate. Consistently, mutations at the $\mathrm{His}^{73}$ and $\mathrm{His}^{77}$ in EcThrRS or their corresponding sites in ScThrRS totally abolish post-transfer editing [42]. However, despite extensive studies for several decades, the detailed hydrolysis mechanism is still undefined in most aaRSs.

\subsubsection{AaRSs with abolished post-transfer editing}

As the last step of aminoacylation accuracy checkpoint, post-transfer editing is considered to be the most economic and efficient mechanism. AaRSs with defective posttransfer editing activity all significantly synthesize mischarged tRNAs. Editing-abolished aaRSs could be artificially achieved by mutating the essential residues, such as the conserved Asp (Asp ${ }^{373}$ in $A a$ LeuRS, Asp ${ }^{419}$ in ScLeuRS, $\mathrm{Asp}^{444}$ in Giardia lamblia LeuRS, etc.) in class Ia synthe- tases [33,83,84], Lys ${ }^{279}$ in E. coli ProRS [28], and $\mathrm{His}^{73}$ and $\mathrm{His}^{77}$ in EcThrRS [42]. Strikingly, there are increasing examples of present-day naturally evolved aaRSs with an abolished post-transfer editing activity. The crucial sites for substrate binding and hydrolysis in the CP1 domain of human mitochondrial LeuRS have been obviously diverged, leading to its failure to edit mis-charged tRNA ${ }^{\text {Leu }}[85,86]$. In $S$. cerevisiae, mitochondrial ThrRS loses the N-terminal domain in evolution, which harbors the editing active site [32]. A similar phenomenon is also observed in S. cerevisiae mitochondrial PheRS without the B3/B4 editing module [87]. Despite the possibility of higher level of selection at the amino acid activation step, the exact mechanism for translational quality control is unclear within the mitochondrion. It is possible that some trans-acting activity exists to remove mis-charged tRNAs but it has been excluded in yeast mitochondrial Tyr-tRNA ${ }^{\text {Phe }}$ hydrolysis [88]. In addition, some bacterial cytoplasm encodes editing-deficient aaRSs which either harbor an impaired editing domain or contains no editing module at all. The most-recently identified instances are the LeuRS and PheRS from some mycoplasma parasites. Both Mycoplasma mobile LeuRS and PheRS are error-prone synthetases, synthesizing Ile-tRNA ${ }^{\text {Leu }}$ and Tyr-tRNA ${ }^{\text {Phe }}$, respectively $[31,89,90]$. Consistently, Mycoplasma mobile has a statistical proteome derived from mistranslation by these mis-charged tRNA species. The evolutionary significance of evolved error-prone aaRSs and the pattern to avoid mistranslational toxicity is undefined. Some organelles or organisms may endure a certain level of mistranslation. In some cases, it may also be associated with the requirement of antigenic diversity or phenotypic plasticity of specific organelle or organism [91].

\section{Additional clearance of mis-charged tRNAs by non-aaRS proteins}

Post-transfer editing activity catalyzed by aaRSs is indispensable for avoiding mistranslation; however, it is not confined to tRNA synthetases [15,92]. Several kinds of freestanding proteins have been identified to be active participants in the translational quality control. Despite the fact that editing active sites are usually conserved among species, the structure, distribution and editing specificity of these trans-acting factors are considerably more divergent throughout evolution [15]. These non-aaRS proteins provide functional redundancy or supplementation of editing catalyzed by aaRSs. Their presence further highlights the crucial importance of maintaining correct formation of cognate aminoacyl-tRNAs.

\subsection{Ala-tRNA ${ }^{\text {Pro }}$ deacylation by ProX}

ProRSs mis-activate non-cognate Ala and form AlatRNA $^{\text {Pro }}$, which is hydrolyzed by the INS domain of bacte- 
rial ProRSs [49]. However, the INS domain is missing from eukaryotic ProRSs [71]. ProX is a widely distributed free-standing protein and homologous to the INS domain [93]. Both bacterial and human ProXs have been shown to edit Ala-tRNA ${ }^{\text {Pro }}$ but fail to hydrolyze Pro-tRNA ${ }^{\text {Pro }}$ and ProRS-formed Cys-tRNA ${ }^{\text {Pro }}[93,94]$. Clostridium sticklandii ProX has been proposed to rely on a conserved His ${ }^{130}$ residue to determine its hydrolysis specificity; however, the corresponding $\mathrm{His}^{126}$ residue of human ProX is not the specificity determinant. In contrast to this amino acid specificity, mis-charged tRNA recognition by ProX is not species-specific and the $\mathrm{CCA}_{76}$ of tRNA plays an important role in recognition [94].

\subsection{Cys-tRNA $^{\text {Pro }}$ deacylation by Ybak}

ProRSs from all three domains of life have the inherent ability to mis-activate Cys and to misacylate Cys onto tRNA $^{\text {Pro }}$, but they lack a Cys-specific editing domain/ activity [95]. Therefore, the double-sieve mechanism seems to be insufficient for removing Cys-tRNA ${ }^{\text {Pro }}$. Another free-standing protein Ybak, which displays significant sequence and structural homology to the INS domain of bacterial ProRS and ProX, is found to hydrolyze Cys-tRNA ${ }^{\text {Pro }}$ by post-transfer editing in trans [96]. Ybak is shown to be a general Cys-tRNA deacylase but preferentially hydrolyzes Cys-tRNA $^{\text {Pro }}$ and Cys-tRNA ${ }^{\text {Cys }}$ [97]. Cys-tRNA ${ }^{\text {Cys }}$ clearance may be avoided because YbaK forms a functional complex with the ProRS-tRNA ${ }^{\text {Pro }}$ complex, preventing Ybak from accessing the Cys-tRNA ${ }^{\text {Cys }}$ synthesized by cysteinyl-tRNA synthetase (CysRS). In addition, the rate of Cys-tRNA ${ }^{\text {Cys }}$ hydrolysis by $\mathrm{YbaK}$ is significantly lower than the rate of Cys-tRNA ${ }^{\text {Cys }}$ synthesis by CysRS [98].

\subsection{Gly/Ser-tRNA ${ }^{\text {Ala }}$ deacylation by AlaX}

In addition to cognate Ala, two non-cognate amino acids Gly and Ser are occasionally mis-activated by AlaRS. Despite the editing domain's ability to clear mis-charged tRNA $^{\mathrm{Ala}} \mathrm{s}$, the mistranslation of Gly/Ser at Ala codons is so acute that an additional layer of checkpoint is required [60]. AlaX, which could be divided into two types based on the presence or absence of C-Ala domain, is encoded in the three domains of life [93]. In mammals, it is expressed as a fused protein with the HSP90 cochaperone p23 homolog in some tissues [99]. The active site for hydrolysis shares homology with the editing domains of AlaRS and ThrRS. Archaeal AlaX has been demonstrated to hydrolyze both Gly-tRNA $^{\text {Ala }}$ and Ser-tRNA ${ }^{\text {Ala }}$ species [93]; however, eukaryotic AlaXs are found to only be able to hydrolyze Ser-tRNA ${ }^{\text {Ala }}[100]$.

\subsection{Ser-tRNA ${ }^{\text {Thr }}$ deacylation by ThrRS-ed}

In bacteria and eukaryotes, the editing domain of ThrRS is located at the N-terminal domain distant from the aminoacylation active site [55]. The N-terminus of most archaeal ThrRSs displays significant sequence divergence from the corresponding editing domain of bacterial ThrRS. Nevertheless, it indeed participates in the editing of Ser-tRNA ${ }^{\text {Thr }}$ with a similar catalytic mechanism to its bacterial counterparts [72]. In these two cases, the editing domain is embedded in the full-length ThrRS. However, several crenarchaeal species contain individual genes encoding the catalytic (ThrRS-cat) and editing domains (ThrRS-ed). The former catalyzes the formation of both Thr-tRNA ${ }^{\text {Thr }}$ and SertRNA $^{\text {Thr }}$ but is unable to cleave Ser-tRNA ${ }^{\text {Thr }}$. In contrast, ThrRS-ed has no aminoacylation activity but evolves the editing activity to hydrolyze the Ser-tRNA ${ }^{\text {Thr }}$ produced by ThrRS-cat. Gene deletion assays indicate that ThrRS-ed is dispensable for growth under standard conditions but is required for normal growth in environments with high concentration of Ser [48]. Whether ThrRS-cat and ThrRS-ed forms an aminoacylation/editing complex and how the mis-charged $\mathrm{tRNA}^{\mathrm{Thr}}$ is transported between two proteins are unclear.

\subsection{D-amino acid-tRNA deacylation by DTD}

D-amino acids are strictly excluded from the protein biosynthesis machinery because of the stereospecificity of aaRS, EF-Tu and the ribosome [101]. However, several aaRSs have been found to load tRNAs with D-amino acids, necessitating D-amino acid-tRNA deacylation activity [102]. DTD is found in the three domains of life and is not specific for tRNA substrates but for a D-amino acid. To date, there are three types of DTDs identified including DTD1 in most bacteria and eukaryotes, DTD2 in archaea and plants, and DTD3 in cyanobacteria [103]. E. coli DTD is a functional homodimer in both solution and structure [104]. It plays a fundamental role in cell viability. Gene inactivation in E. coli or in Saccharomyces cerevisiae exacerbates cell growth inhibition by several D-amino acids [102]. Interestingly, the crystal structure of some archaea-specific editing domain of ThrRS (such as Pyrococcus abyssi ThrRS) has revealed it shares a marked structural similarity with DTD, implying a functional connection and/or evolutionary relationship between these two domains [73].

\section{Mis-charged tRNA species necessary and es- sential for the cell}

Indeed, mis-charged tRNAs should be rejected from the protein biosynthesis machinery for the correct transduction of genetic code. The mis-charged tRNA species discussed above are hydrolyzed by different means to avoid harm to the cell. However, some mis-chargered tRNA species are far from harmful but necessary and essential for the flow of genetic code or for countering specific stresses. These 
mis-charged tRNAs are either intermediates of a specific pathway for correctly charged tRNA formation or directly utilized by the ribosome.

\subsection{Glu-tRNA ${ }^{\text {Gln }}$}

All known archaea (e.g., Methanothermobacter thermautotrophicus), most bacteria (e.g., Bacillus subtilis), chloroplast, and mitochondria lack GlnRS to synthesize the GlntRNA $^{\text {Gln }}$ [105-108]. Instead, in these organelles or organisms, GluRS is non-discriminating (ND-GluRS), which means that it can also recognize tRNA ${ }^{\text {Gln }}$ other than its normal substrate tRNA ${ }^{\text {Glu }}$ [109-111]. In contrast, its counterparts in some bacteria (e.g., E. coli) and all eukaryotes (e.g., human beings) encode a discriminating GluRS unable to recognize and charge tRNA ${ }^{\text {Gln }}$. In these cases, GlnRSs are also encoded [7]. The produced Glu-tRNA ${ }^{\text {Gln }}$ is not transported to the EF-Tu/ribosome machinery. Instead, it is further processed by an amidotransferase activity (GatCAB in bacteria or GatDE in archaea) $[112,113]$. This is realized in the stable complex (termed as transaminosome) formed by both the ND-GluRS and the amidotransferase [114].

\subsection{Asp-tRNA ${ }^{\text {Asn }}$}

Most bacteria (e.g., Helicobacter pylori) and archaea (e.g., $M$. thermautotrophicus) encode a non-discriminating aspartyl-tRNA synthetase (ND-AspRS), but no aspariginyl-tRNA synthetase (AsnRS) activity [115]. Asp-tRNA ${ }^{\text {Asp }}$ for the ribosomal protein biosynthesis is produced by the ND-AspRS [116]. In addition, ND-AspRS also ligates Asp with non-cognate tRNA $^{\text {Asn }}$ to form mis-charged Asp-tRNA $^{\text {Asn }}$ [117]. Asp-tRNA ${ }^{\text {Asn }}$ is then converted into Asn-tRNA $^{\text {Asn }}$ by the GatCAB [118-121].

\subsection{Ser/Sep-tRNA ${ }^{\mathrm{Sec}}$}

Selenocysteine (2-selenoalanine, $\mathrm{Sec}$ ) is designated as the 21 st proteinaceous amino acid. The in-frame nonsense codon UGA of the specific mRNA of Sec-decoding organisms specifies the incorporation of Sec in bacteria, archaea, and eukaryotes [122]. A structurally unique tRNA (tRNA $\left.{ }^{\mathrm{Sec}}\right)$ functions as the key player in the Sec biosynthesis and the decoding process at the ribosome. The most strikingly features of this tRNA are the UCA anti-codon used to decode the UGA codon, the elongated acceptor stem (nine base pairs), a small D-loop, and a long variable stem and loop [122]. Sec formation and incorporation in the proteome is very important for organisms as reflected by the embryonic lethal phenotype of tRNA ${ }^{\text {Sec }}$ knockout mouse [123]. In addition, Sec is present in the active sites of enzymes involved in removing reactive oxidative species and in thyroid hormone activation $[124,125]$. Sec-tRNA ${ }^{\text {Sec }}$ formation is a multi-step process involving several proteins and co-factors. The tRNA ${ }^{\mathrm{Sec}}$ is first aminoacylated by the SerRS to form the mis-charged Ser-tRNA ${ }^{\mathrm{Sec}}$ [126]. In bacteria, a protein called selenocysteine synthase (SelA) directly converts the Ser-tRNA $^{\text {Sec }}$ into cognate Sec-tRNA ${ }^{\text {Sec }}$ [127]. An alternative pathway exists in archaea and eukaryotes. The Ser moiety of mis-charged tRNA $^{\mathrm{Sec}}$ is first phosphorylated by O-phosphoseryl-tRNA ${ }^{\text {Sec }}$ kinase (PSTK) [128], converting Ser-tRNA $^{\text {Sec }}$ to O-phosphoseryl-tRNA ${ }^{\text {Sec }}\left(\right.$ Sep-tRNA $\left.{ }^{\text {Sec }}\right)$, which is subsequently converted to Sec-tRNA ${ }^{\text {Sec }}$ by an additional enzyme, O-phosphoseryl-tRNA:selenocysteinyltRNA synthase $(\mathrm{SepSecS})$ [129]. Sec-tRNA ${ }^{\mathrm{Sec}}$ is then transferred to the ribosome to decode the UGA codon which possesses a hairpin structure and SECIS element [130].

\subsection{Sep-tRNA ${ }^{\text {Cys }}$}

Several archaea (e.g., Methanocaldococcus jannaschii) lack CysRS, the essential enzyme that provides Cys-tRNA ${ }^{\mathrm{Cys}}$ for translation in most organisms. However, Cys-tRNA ${ }^{\text {Cys }}$ formation is performed by an alternative and more complicated pathway [131]. tRNA ${ }^{\text {Cys }}$ is first esterified with the O-phosphoserine (Sep) by a class II-type O-phosphoseryl-tRNA synthetase (SepRS) to form mis-charged Sep-tRNA ${ }^{\text {Cys }}$ [132]. Sep-tRNA ${ }^{\text {Cys }}$ is the intermediate and subsequently converted to Cys-tRNA ${ }^{\text {Cys }}$ by the Sep-tRNA:Cys-tRNA synthase (SepCysS) in the presence of a sulfur donor [133,134].

\subsection{Leu-tRNA Ler $^{\text {Ser }}$}

In most species, the CUG codon determines the incorporation of Leu at the ribosome. However, in several species of the genus Candida and Debaryomyces, the CUG codons are decoded as both Ser and Leu [135]. A chimeric tRNA, tRNA $^{\mathrm{Ser}}(\mathrm{CAG})$ is the key molecule for this genetic code reassignment [136]. This tRNA has the feature of both tRNA $^{\text {Leu }}$ and tRNA $^{\text {Ser }}$ aminoacylation identity elements with the anti-codon (CAG) matching the CUG codon. In the cytoplasm of these species, nearly $3 \%$ of total tRNA ${ }^{\text {Ser }}$ (CAG) is ligated with Leu (Leu-tRNA ${ }^{\text {Ser }}$ ) by LeuRS with the remaining tRNA ${ }^{\text {Ser }}(\mathrm{CAG})$ charged with Ser $\left(\right.$ Ser-tRNA $\left.{ }^{\text {Ser }}\right)$ by SerRS [137]. Consistently, C. albicans decodes CUG codons ambiguously and tolerates partial reversion of CUG codon from Ser back to Leu on a genome-wide scale. Our recent study also reveals that only eukaryotic LeuRSs but not archaeal or bacterial LeuRSs could aminoacylate the tRNA $^{\mathrm{Ser}}(\mathrm{CAG})$, indicating that the C-terminal domain of eukaryotic LeuRSs is responsible for tRNA recognition and also suggesting that one can artificially modify the genetic code property of an eukaryote by simply introducing the tRNA $^{\text {Ser }}$ (CAG) [138]. This ambiguity is not meaningless, harmful, but instead is used to generate important phenotypic diversity owing to the expanded proteome [91].

\subsection{Met misacylated tRNAs}

Recent studies have demonstrated that in both yeast and 
mammalian cells, methionyl-tRNA synthetase (MetRS) can mis-charge non-methionyl tRNAs at frequencies of about $10 \%$ in vivo $[138,139]$. The identity elements for this kind of mis-methionylation reside, at least partly, in the anticodon region of non-methionyl tRNAs [140]. The produced Met mis-acylated tRNAs are readily accommodated and used at the ribosome and some Met mis-incoporation sites have been identified by mass spectrometry [138]. The biological significance of this type of mis-charging remains undefined. It is suggested that Met mis-incorporation under stress conditions may contribute to protecting the produced protein against the stress. Indeed, exposing cells to innate immune (e.g., virus) and chemically triggered oxidative stress (e.g., arsenite, $\mathrm{H}_{2} \mathrm{O}_{2}$, etc.) promote Met-misaclyation at a higher level [138].

\section{Concluding remarks}

tRNA is one of the most important molecules in the protein biosynthesis pathway. The correct ligation of amino acids and tRNAs by aaRSs is critical for the genetic code transduction for most organisms. In spite of finding of editing for several decades, the detailed and dynamic editing mechanism still needs to be defined. Future work should be directed to solve the connection of aminoacylation, editing and cellular dysfunction, thus improving our understanding of the physiological significance of editing and facilitating disease diagnosis and treatment. In addition, because of the essential function, wide distribution, sequence divergence and two active sites (aminoacylation and editing) of some aaRSs from human and disease-causing organisms, aaRSs are ideal target molecules for drug design and development. Therefore, aaRS aminoacylation and/or editing domaintargeted screening of small inhibitors should be expanded to enlarge and enrich the resource of lead components or drugs from either naturally or chemically synthesized agents.

We thank Dr. Joseph Lee (Harvard Medical School, Boston, USA) for critically reading the manuscript. We also gratefully acknowledge the support of SA-SIBS scholarship program. This work was supported by the National Natural Science Foundation of China (31270852, 31000355) and the National Key Basic Research Program of China (2012CB911000).

1 Ibba M, Söll D. Aminoacyl-tRNA synthesis. Annu Rev Biochem, 2000, 69: 617-650

2 Giege R. Toward a more complete view of tRNA biology. Nat Struct Mol Biol, 2008, 15: 1007-1014

3 Francklyn C S, Minajigi A. tRNA as an active chemical scaffold for diverse chemical transformations. FEBS Lett, 2010, 584: 366-375

4 Mei Y, Stonestrom A, Hou Y M, et al. Apoptotic regulation and tRNA. Protein Cell, 2010, 1: 795-801

5 Dare K, Ibba M. Roles of tRNA in cell wall biosynthesis. Wiley Interdiscip Rev RNA, 2012, 3: 247-264

6 Kleiman L, Jones C P, Musier-Forsyth K. Formation of the tRNALys packaging complex in HIV-1. FEBS Lett, 2010, 584: 359-365

7 Yuan J, Sheppard K, Söll D. Amino acid modifications on tRNA. Acta Biochim Biophys Sin (Shanghai), 2008, 40: 539-553
8 Sobala A, Hutvagner G. Transfer RNA-derived fragments: origins, processing, and functions. Wiley Interdiscip Rev RNA, 2011, 2: 853-862

9 Woese C R, Olsen G J, Ibba M, et al. Aminoacyl-tRNA synthetases, the genetic code, and the evolutionary process. Microbiol Mol Biol Rev, 2000, 64: 202-236

10 Eriani G, Delarue M, Poch O, et al. Partition of tRNA synthetases into two classes based on mutually exclusive sets of sequence motifs. Nature, 1990, 347: 203-206

11 Cusack S, Berthet-Colominas C, Hartlein M, et al. A second class of synthetase structure revealed by X-ray analysis of Escherichia coli seryl-tRNA synthetase at 2.5 A. Nature, 1990, 347: 249-255

12 Baldwin A N, Berg P. Transfer ribonucleic acid-induced hydrolysis of valyladenylate bound to isoleucyl ribonucleic acid synthetase. J Biol Chem, 1966, 241: 839-845

13 Fersht A R. Editing mechanisms in protein synthesis. Rejection of valine by the isoleucyl-tRNA synthetase. Biochemistry, 1977, 16: 1025-1030

14 Fersht A R, Dingwall C. Evidence for the double-sieve editing mechanism in protein synthesis. Steric exclusion of isoleucine by valyl-tRNA synthetases. Biochemistry, 1979, 18: 2627-2631

15 Ling J, Reynolds N, Ibba M. Aminoacyl-tRNA synthesis and translational quality control. Annu Rev Microbiol, 2009, 63: 61-78

16 Ibba M, Söll D. Quality control mechanisms during translation. Science, 1999, 286: 1893-1897

17 Doring V, Mootz H D, Nangle L A, et al. Enlarging the amino acid set of Escherichia coli by infiltration of the valine coding pathway. Science, 2001, 292: 501-504

18 Bacher J M, de Crecy-Lagard V, Schimmel P R. Inhibited cell growth and protein functional changes from an editing-defective tRNA synthetase. Proc Natl Acad Sci USA, 2005, 102: 1697-1701

19 Nangle L A, Motta C M, Schimmel P. Global effects of mistranslation from an editing defect in mammalian cells. Chem Biol, 2006, 13: 1091-1100

20 Lee J W, Beebe K, Nangle L A, et al. Editing-defective tRNA synthetase causes protein misfolding and neurodegeneration. Nature, 2006, 443: 50-55

21 Schimmel P. Development of tRNA synthetases and connection to genetic code and disease. Protein Sci, 2008, 17: 1643-1652

22 Giege R, Sissler M, Florentz C. Universal rules and idiosyncratic features in tRNA identity. Nucleic Acids Res, 1998, 26: 5017-5035

23 Schmidt E, Schimmel P. Mutational isolation of a sieve for editing in a transfer RNA synthetase. Science, 1994, 264: 265-267

24 Zhu B, Yao P, Tan M, et al. tRNA-independent pretransfer editing by class I leucyl-tRNA synthetase. J Biol Chem, 2009, 284: 34183424

25 Banerjee R, Chen S, Dare K, et al. tRNAs: cellular barcodes for amino acids. FEBS Lett, 2010, 584: 387-395

26 Gruic-Sovulj I, Uter N, Bullock T, et al. tRNA-dependent aminoacyl-adenylate hydrolysis by a nonediting class I aminoacyltRNA synthetase. J Biol Chem, 2005, 280: 23978-23986

27 Hati S, Ziervogel B, Sternjohn J, et al. Pre-transfer editing by class II prolyl-tRNA synthetase: role of aminoacylation active site in "selective release" of noncognate amino acids. J Biol Chem, 2006, 281: 27862-27872

28 Splan K E, Ignatov M E, Musier-Forsyth K. Transfer RNA modulates the editing mechanism used by class II prolyl-tRNA synthetase. J Biol Chem, 2008, 283: 7128-7134

29 Zhou X L, Ruan Z R, Huang Q, et al. Translational fidelity maintenance preventing Ser mis-incorporation at Thr codon in protein from eukaryote. Nucleic Acids Res, 2013, 41: 302-314

30 Gruic-Sovulj I, Rokov-Plavec J, Weygand-Durasevic I. Hydrolysis of non-cognate aminoacyl-adenylates by a class II aminoacyl-tRNA synthetase lacking an editing domain. FEBS Lett, 2007, 581: 5110-5114

31 Tan M, Yan W, Liu R J, et al. A naturally occurring nonapeptide functionally compensates for the CP1 domain of leucyl-tRNA synthetase to modulate aminoacylation activity. Biochem J, 2012, 443: 477-484 
32 Ling J, Peterson K M, Simonovic I, et al. The mechanism of pre-transfer editing in yeast mitochondrial threonyl-tRNA synthetase. J Biol Chem, 2012, 287: 28518-28525

33 Tan M, Zhu B, Zhou X L, et al. tRNA-dependent pre-transfer editing by prokaryotic leucyl-tRNA synthetase. J Biol Chem, 2010, 285: 3235-3244

34 Nordin B E, Schimmel P. Transiently misacylated tRNA is a primer for editing of misactivated adenylates by class I aminoacyl-tRNA synthetases. Biochemistry, 2003, 42: 12989-12997

35 Bishop A C, Beebe K, Schimmel P R. Interstice mutations that block site-to-site translocation of a misactivated amino acid bound to a class I tRNA synthetase. Proc Natl Acad Sci USA, 2003, 100: 490494

36 Lincecum T L Jr., Tukalo M, Yaremchuk A, et al. Structural and mechanistic basis of pre- and posttransfer editing by leucyl-tRNA synthetase. Mol Cell, 2003, 11: 951-963

37 Fukunaga R, Yokoyama S. Structural basis for substrate recognition by the editing domain of isoleucyl-tRNA synthetase. J Mol Biol, 2006, 359: 901-912

38 Nomanbhoy T K, Hendrickson T L, Schimmel P. Transfer RNAdependent translocation of misactivated amino acids to prevent errors in protein synthesis. Mol Cell, 1999, 4: 519-528

39 Silvian L F, Wang J, Steitz T A. Insights into editing from an ile-tRNA synthetase structure with tRNAile and mupirocin. Science, 1999, 285: 1074-1077

40 Fukai S, Nureki O, Sekine S, et al. Structural basis for double-sieve discrimination of L-valine from $\mathrm{L}$-isoleucine and L-threonine by the complex of tRNA(Val) and valyl-tRNA synthetase. Cell, 2000, 103: 793-803

41 Dulic M, Cvetesic N, Perona J J, et al. Partitioning of tRNAdependent editing between pre- and post-transfer pathways in class I aminoacyl-tRNA synthetases. J Biol Chem, 2010, 285: 23799-23809

42 Dock-Bregeon A C, Rees B, Torres-Larios A, et al. Achieving error-free translation; the mechanism of proofreading of threonyltRNA synthetase at atomic resolution. Mol Cell, 2004, 16: 375-386

43 Zhou X L, Du D H, Tan M, et al. Role of tRNA amino acidaccepting end in aminoacylation and its quality control. Nucleic Acids Res, 2011, 39: 8857-8868

44 Tan M, Zhu B, Liu R J, et al. Interdomain communication modulates the tRNA-dependent pre-transfer editing of leucyl-tRNA synthetase. Biochem J, 2013, 449: 123-131

45 Eldred E W, Schimmel P R. Rapid deacylation by isoleucyl transfer ribonucleic acid synthetase of isoleucine-specific transfer ribonucleic acid aminoacylated with valine. J Biol Chem, 1972, 247: 2961-2964

46 Nordin B E, Schimmel P. Plasticity of recognition of the 3'-end of mischarged tRNA by class I aminoacyl-tRNA synthetases. J Biol Chem, 2002, 277: 20510-20517

47 Zhao M W, Zhu B, Hao R, et al. Leucyl-tRNA synthetase from the ancestral bacterium Aquifex aeolicus contains relics of synthetase evolution. EMBO J, 2005, 24: 1430-1439

48 Korencic D, Ahel I, Schelert J, et al. A freestanding proofreading domain is required for protein synthesis quality control in Archaea. Proc Natl Acad Sci USA, 2004, 101: 10260-10265

49 Wong F C, Beuning P J, Silvers C, et al. An isolated class II aminoacyl-tRNA synthetase insertion domain is functional in amino acid editing. J Biol Chem, 2003, 278: 52857-52864

50 Beebe K, Ribas De Pouplana L, Schimmel P. Elucidation of tRNAdependent editing by a class II tRNA synthetase and significance for cell viability. EMBO J, 2003, 22: 668-675

51 Roy $\mathrm{H}$, Ling J, Irnov $\mathrm{M}$, et al. Post-transfer editing in vitro and in vivo by the beta subunit of phenylalanyl-tRNA synthetase. EMBO J, 2004, 23: 4639-4648

52 Ling J, So B R, Yadavalli S S, et al. Resampling and editing of mischarged tRNA prior to translation elongation. Mol Cell, 2009, 33: 654-660

53 Dock-Bregeon A, Sankaranarayanan R, Romby P, et al. Transfer RNA-mediated editing in threonyl-tRNA synthetase. The class II solution to the double discrimination problem. Cell, 2000, 103: $877-884$
54 Nureki O, Vassylyev D G, Tateno M, et al. Enzyme structure with two catalytic sites for double-sieve selection of substrate. Science, 1998, 280: 578-582

55 Sankaranarayanan R, Dock-Bregeon A C, Romby P, et al. The structure of threonyl-tRNA synthetase-tRNA(Thr) complex enlightens its repressor activity and reveals an essential zinc ion in the active site. Cell, 1999, 97: 371-381

56 Fukunaga R, Yokoyama S. Aminoacylation complex structures of leucyl-tRNA synthetase and tRNALeu reveal two modes of discriminator-base recognition. Nat Struct Mol Biol, 2005, 12: 915922

57 Tukalo M, Yaremchuk A, Fukunaga R, et al. The crystal structure of leucyl-tRNA synthetase complexed with tRNALeu in the posttransfer-editing conformation. Nat Struct Mol Biol, 2005, 12: 923930

58 Palencia A, Crepin T, Vu M T, et al. Structural dynamics of the aminoacylation and proofreading functional cycle of bacterial leucyl-tRNA synthetase. Nat Struct Mol Biol, 2012, 19: 677-684

59 Beebe K, Mock M, Merriman E, et al. Distinct domains of tRNA synthetase recognize the same base pair. Nature, 2008, 451: 90-93

60 Guo M, Chong Y E, Beebe K, et al. The C-Ala domain brings together editing and aminoacylation functions on one tRNA. Science, 2009, 325: 744-747

61 Zhou X L, Yao P, Ruan L L, et al. A unique insertion in the CP1 domain of Giardia lamblia leucyl-tRNA synthetase. Biochemistry, 2009, 48: 1340-1347

62 Fukunaga R, Yokoyama S. Crystal structure of leucyl-tRNA synthetase from the archaeon Pyrococcus horikoshii reveals a novel editing domain orientation. J Mol Biol, 2005, 346: 57-71

63 Fukunaga R, Fukai S, Ishitani R, et al. Crystal structures of the CP1 domain from Thermus thermophilus isoleucyl-tRNA synthetase and its complex with L-valine. J Biol Chem, 2004, 279: 8396-8402

64 Fukunaga R, Yokoyama S. Structural basis for non-cognate amino acid discrimination by the valyl-tRNA synthetase editing domain. J Biol Chem, 2005, 280: 29937-29945

65 Zhu B, Zhao M W, Eriani G, et al. A present-day aminoacyl-tRNA synthetase with ancestral editing properties. RNA, 2007, 13: 15-21

66 Liu R J, Tan M, Du D H, et al. Peripheral insertion modulates the editing activity of the isolated CP1 domain of leucyl-tRNA synthetase. Biochem J, 2011, 440: 217-227

67 Lin L, Hale S P, Schimmel P. Aminoacylation error correction. Nature, 1996, 384: 33-34

68 Chen J F, Guo N N, Li T, et al. CP1 domain in Escherichia coli leucyl-tRNA synthetase is crucial for its editing function. Biochemistry, 2000, 39: 6726-6731

69 Zhou X L, Wang E D. Two tyrosine residues outside the editing active site in Giardia lamblia leucyl-tRNA synthetase are essential for the post-transfer editing. Biochem Biophys Res Commun, 2009, 386: 510-515

70 Rock F L, Mao W, Yaremchuk A, et al. An antifungal agent inhibits an aminoacyl-tRNA synthetase by trapping tRNA in the editing site. Science, 2007, 316: 1759-1761

71 Crepin T, Yaremchuk A, Tukalo M, et al. Structures of two bacterial prolyl-tRNA synthetases with and without a cis-editing domain. Structure, 2006, 14: 1511-1525

72 Hussain T, Kruparani S P, Pal B, et al. Post-transfer editing mechanism of a D-aminoacyl-tRNA deacylase-like domain in threonyl-tRNA synthetase from Archaea. EMBO J, 2006, 25: 4152-4162

73 Dwivedi S, Kruparani S P, Sankaranarayanan R. A D-amino acid editing module coupled to the translational apparatus in Archaea. Nat Struct Mol Biol, 2005, 12: 556-557

74 Hussain T, Kamarthapu V, Kruparani S P, et al. Mechanistic insights into cognate substrate discrimination during proofreading in translation. Proc Natl Acad Sci USA, 2010, 107: 22117-22121

75 Roy H, Ibba M. Phenylalanyl-tRNA synthetase contains a dispensable RNA-binding domain that contributes to the editing of noncognate aminoacyl-tRNA. Biochemistry, 2006, 45: 9156-9162

76 Ling J, Yadavalli S S, Ibba M. Phenylalanyl-tRNA synthetase 
editing defects result in efficient mistranslation of phenylalanine codons as tyrosine. RNA, 2007, 13: 1881-1886

77 Tardif K D, Horowitz J. Transfer RNA determinants for translational editing by Escherichia coli valyl-tRNA synthetase. Nucleic Acids Res, 2002, 30: 2538-2545

78 Du X, Wang E D. Tertiary structure base pairs between D- and TpsiC-loops of Escherichia coli tRNA(Leu) play important roles in both aminoacylation and editing. Nucleic Acids Res, 2003, 31: 2865-2872

79 Yao P, Zhu B, Jaeger S, et al. Recognition of tRNALeu by Aquifex aeolicus leucyl-tRNA synthetase during the aminoacylation and editing steps. Nucleic Acids Res, 2008, 36: 2728-2738

80 Hale S P, Auld D S, Schmidt E, et al. Discrete determinants in transfer RNA for editing and aminoacylation. Science, 1997, 276: 1250-1252

81 Huang Q, Yao P, Eriani G, et al. In vivo identification of essential nucleotides in tRNALeu to its functions by using a constructed yeast tRNALeu knockout strain. Nucleic Acids Res, 2012, 40: 1046310477

$82 \mathrm{Xu} \mathrm{M} \mathrm{G,} \mathrm{Li} \mathrm{J,} \mathrm{Du} \mathrm{X,} \mathrm{et} \mathrm{al.} \mathrm{Groups} \mathrm{on} \mathrm{the} \mathrm{side} \mathrm{chain} \mathrm{of} \mathrm{T252} \mathrm{in}$ Escherichia coli leucyl-tRNA synthetase are important for discrimination of amino acids and cell viability. Biochem Biophys Res Commun, 2004, 318: 11-16

83 Yao P, Zhou X L, He R, et al. Unique residues crucial for optimal editing in yeast cytoplasmic Leucyl-tRNA synthetase are revealed by using a novel knockout yeast strain. J Biol Chem, 2008, 283: 22591-22600

84 Zhou X L, Tan M, Wang M, et al. Post-transfer editing by a eukaryotic leucyl-tRNA synthetase resistant to the broad-spectrum drug AN2690. Biochem J, 2010, 430: 325-333

85 Lue S W, Kelley S O. An aminoacyl-tRNA synthetase with a defunct editing site. Biochemistry, 2005, 44: 3010-3016

86 Yao Y N, Wang L, Wu X F, et al. Human mitochondrial leucyltRNA synthetase with high activity produced from Escherichia coli. Protein Expr Purif, 2003, 30: 112-116

87 Roy H, Ling J, Alfonzo J, et al. Loss of editing activity during the evolution of mitochondrial phenylalanyl-tRNA synthetase. J Biol Chem, 2005, 280: 38186-38192

88 Reynolds N M, Ling J, Roy H, et al. Cell-specific differences in the requirements for translation quality control. Proc Natl Acad Sci USA, 2010, 107: 4063-4068

89 Li L, Boniecki M T, Jaffe J D, et al. Naturally occurring aminoacyl-tRNA synthetases editing-domain mutations that cause mistranslation in Mycoplasma parasites. Proc Natl Acad Sci USA, 2011, 108: 9378-9383

90 Yadavalli S S, Ibba M. Selection of tRNA charging quality control mechanisms that increase mistranslation of the genetic code. Nucleic Acids Res, 2013, 41: 1104-1112

91 Gomes A C, Miranda I, Silva R M, et al. A genetic code alteration generates a proteome of high diversity in the human pathogen Candida albicans. Genome Biol, 2007, 8: R206

92 Reynolds N M, Lazazzera B A, Ibba M. Cellular mechanisms that control mistranslation. Nat Rev Microbiol, 2010, 8: 849-856

93 Ahel I, Korencic D, Ibba M, et al. Trans-editing of mischarged tRNAs. Proc Natl Acad Sci USA, 2003, 100: 15422-15427

94 Ruan L L, Zhou X L, Tan M, et al. Human cytoplasmic ProX edits mischarged tRNAPro with amino acid but not tRNA specificity. Biochem J, 2013, 450: 243-252

95 Ahel I, Stathopoulos C, Ambrogelly A, et al. Cysteine activation is an inherent in vitro property of prolyl-tRNA synthetases. J Biol Chem, 2002, 277: 34743-34748

96 An S, Musier-Forsyth K. Trans-editing of Cys-tRNAPro by Haemophilus influenzae YbaK protein. J Biol Chem, 2004, 279: 42359-42362

97 Ruan B, Söll D. The bacterial YbaK protein is a Cys-tRNAPro and Cys-tRNA Cys deacylase. J Biol Chem, 2005, 280: 25887-25891

98 An S, Musier-Forsyth K. Cys-tRNA(Pro) editing by Haemophilus influenzae YbaK via a novel synthetase.YbaK.tRNA ternary complex. J Biol Chem, 2005, 280: 34465-34472
99 Nawaz M H, Merriman E, Yang X L, et al. p23H implicated as cis/trans regulator of AlaXp-directed editing for mammalian cell homeostasis. Proc Natl Acad Sci USA, 2011, 108: 2723-2728

100 Guo M, Chong Y E, Shapiro R, et al. Paradox of mistranslation of serine for alanine caused by AlaRS recognition dilemma. Nature, 2009, 462: 808-812

101 Yamane T, Miller D L, Hopfield J J. Discrimination between D- and L-tyrosyl transfer ribonucleic acids in peptide chain elongation. Biochemistry, 1981, 20: 7059-7064

102 Soutourina J, Plateau P, Blanquet S. Metabolism of D-aminoacyltRNAs in Escherichia coli and Saccharomyces cerevisiae cells. J Biol Chem, 2000, 275: 32535-32542

103 Wydau S, van der Rest G, Aubard C, et al. Widespread distribution of cell defense against D-aminoacyl-tRNAs. J Biol Chem, 2009, 284: 14096-14104

104 Ferri-Fioni M L, Schmitt E, Soutourina J, et al. Structure of crystalline D-Tyr-tRNA(Tyr) deacylase. A representative of a new class of tRNA-dependent hydrolases. J Biol Chem, 2001, 276: 47285-47290

105 Tumbula D L, Becker H D, Chang W Z, et al. Domain-specific recruitment of amide amino acids for protein synthesis. Nature, 2000, 407: 106-110

106 Nagao A, Suzuki T, Katoh T, et al. Biogenesis of glutaminyl-mt tRNAGln in human mitochondria. Proc Natl Acad Sci USA, 2009, 106: 16209-16214

107 Frechin M, Senger B, Braye M, et al. Yeast mitochondrial Gln-tRNA(Gln) is generated by a GatFAB-mediated transamidation pathway involving Arc1p-controlled subcellular sorting of cytosolic GluRS. Genes Dev, 2009, 23: 1119-1130

108 Pujol C, Bailly M, Kern D, et al. Dual-targeted tRNA-dependent amidotransferase ensures both mitochondrial and chloroplastic Gln-tRNAGln synthesis in plants. Proc Natl Acad Sci USA, 2008, 105: 6481-6485

109 Lapointe J, Duplain L, Proulx M. A single glutamyl-tRNA synthetase aminoacylates tRNAGlu and tRNAGln in Bacillus subtilis and efficiently misacylates Escherichia coli tRNAGln1 in vitro. J Bacteriol, 1986, 165: 88-93

110 Schulze J O, Masoumi A, Nickel D, et al. Crystal structure of a non-discriminating glutamyl-tRNA synthetase. J Mol Biol, 2006, 361: 888-897

111 Guo L T, Helgadottir S, Söll D, et al. Rational design and directed evolution of a bacterial-type glutaminyl-tRNA synthetase precursor. Nucleic Acids Res, 2012, 40: 7967-7974

112 Nakamura A, Yao M, Chimnaronk S, et al. Ammonia channel couples glutaminase with transamidase reactions in GatCAB. Science, 2006, 312: 1954-1958

113 Oshikane H, Sheppard K, Fukai S, et al. Structural basis of RNAdependent recruitment of glutamine to the genetic code. Science, 2006, 312: 1950-1954

114 Ito T, Yokoyama S. Two enzymes bound to one transfer RNA assume alternative conformations for consecutive reactions. Nature, 2010, 467: 612-616

115 Min B, Pelaschier J T, Graham D E, et al. Transfer RNA-dependent amino acid biosynthesis: an essential route to asparagine formation. Proc Natl Acad Sci USA, 2002, 99: 2678-2683

116 Charron $\mathrm{C}$, Roy $\mathrm{H}$, Blaise $\mathrm{M}$, et al. Non-discriminating and discriminating aspartyl-tRNA synthetases differ in the anticodonbinding domain. EMBO J, 2003, 22: 1632-1643

117 Min B, Kitabatake M, Polycarpo C, et al. Protein synthesis in Escherichia coli with mischarged tRNA. J Bacteriol, 2003, 185: 3524-3526

118 Namgoong S, Sheppard K, Sherrer R L, et al. Co-evolution of the archaeal tRNA-dependent amidotransferase GatCAB with tRNA(Asn). FEBS Lett, 2007, 581: 309-314

119 Sheppard K, Söll D. On the evolution of the tRNA-dependent amidotransferases, GatCAB and GatDE. J Mol Biol, 2008, 377: 831-844

120 Bailly M, Blaise M, Lorber B, et al. The transamidosome: a dynamic ribonucleoprotein particle dedicated to prokaryotic tRNA-dependent 
asparagine biosynthesis. Mol Cell, 2007, 28: 228-239

121 Blaise M, Bailly M, Frechin M, et al. Crystal structure of a transferribonucleoprotein particle that promotes asparagine formation. EMBO J, 2010, 29: 3118-3129

122 Commans S, Bock A. Selenocysteine inserting tRNAs: an overview. FEMS Microbiol Rev, 1999, 23: 335-351

123 Bosl M R, Takaku K, Oshima M, et al. Early embryonic lethality caused by targeted disruption of the mouse selenocysteine tRNA gene (Trsp). Proc Natl Acad Sci USA, 1997, 94: 5531-5534

124 Kryukov G V, Castellano S, Novoselov S V, et al. Characterization of mammalian selenoproteomes. Science, 2003, 300: 1439-1443

125 Rayman M P. The importance of selenium to human health. Lancet, 2000, 356: 233-241

126 Ambrogelly A, Palioura S, Söll D. Natural expansion of the genetic code. Nat Chem Biol, 2007, 3: 29-35

127 Forchhammer K, Leinfelder W, Boesmiller K, et al. Selenocysteine synthase from Escherichia coli. Nucleotide sequence of the gene (selA) and purification of the protein. J Biol Chem, 1991, 266: 6318-6323

128 Carlson B A, Xu X M, Kryukov G V, et al. Identification and characterization of phosphoseryl-tRNA[Ser]Sec kinase. Proc Natl Acad Sci USA, 2004, 101: 12848-12853

129 Palioura S, Sherrer R L, Steitz T A, et al. The human SepSecStRNASec complex reveals the mechanism of selenocysteine formation. Science, 2009, 325: 321-325

130 Zinoni F, Heider J, Bock A. Features of the formate dehydrogenase mRNA necessary for decoding of the UGA codon as selenocysteine. Proc Natl Acad Sci USA, 1990, 87: 4660-4664

131 Sauerwald A, Zhu W, Major T A, et al. RNA-dependent cysteine biosynthesis in Archaea. Science, 2005, 307: 1969-1972

132 Kamtekar S, Hohn M J, Park H S, et al. Toward understanding
phosphoseryl-tRNACys formation: the crystal structure of Methanococcus maripaludis phosphoseryl-tRNA synthetase. Proc Natl Acad Sci USA, 2007, 104: 2620-2625

133 Liu Y, Dos Santos P C, Zhu X, et al. Catalytic mechanism of Sep-tRNA:Cys-tRNA synthase: sulfur transfer is mediated by disulfide and persulfide. J Biol Chem, 2012, 287: 5426-5433

134 O'Donoghue P, Sethi A, Woese C R, et al. The evolutionary history of Cys-tRNACys formation. Proc Natl Acad Sci USA, 2005, 102: 19003-19008

135 Moura G R, Paredes J A, Santos M A. Development of the genetic code: insights from a fungal codon reassignment. FEBS Lett, 2010, 584: 334-341

136 Santos M A, Perreau V M, Tuite M F. Transfer RNA structural change is a key element in the reassignment of the CUG codon in Candida albicans. EMBO J, 1996, 15: 5060-5068

137 Suzuki T, Ueda T, Watanabe K. The 'polysemous' codon-a codon with multiple amino acid assignment caused by dual specificity of tRNA identity. EMBO J, 1997, 16: 1122-1134

138 Zhou X L, Fang Z P, Ruan Z R, et al. Aminoacylation and translational quality control strategy employed by leucyl-tRNA synthetase from a human pathogen with genetic code ambiguity. Nucleic Acids Res, 2013, doi: 10.1093/nar/gkt741

139 Netzer N, Goodenbour J M, David A, et al. Innate immune and chemically triggered oxidative stress modifies translational fidelity. Nature, 2009, 462: 522-526

140 Wiltrout E, Goodenbour J M, Frechin M, et al. Misacylation of tRNA with methionine in Saccharomyces cerevisiae. Nucleic Acids Res, 2012, 40: 10494-10506

141 Jones T E, Alexander R W, Pan T. Misacylation of specific nonmethionyl tRNAs by a bacterial methionyl-tRNA synthetase. Proc Natl Acad Sci USA, 2011, 108: 6933-6938

Open Access This article is distributed under the terms of the Creative Commons Attribution License which permits any use, distribution, and reproduction in any medium, provided the original author(s) and source are credited. 